\title{
Cooperative Sensing with Adaptive Sensing Ranges in Cognitive Radio Ad-Hoc Networks
}

\author{
Jongwon Shim \\ Department of Computer Science \\ Oklahoma State University \\ Stillwater, Oklahoma 74078 \\ Email: jongwon@cs.okstate.edu
}

\author{
Qi Cheng \\ School of Electrical and Computer Engineering \\ Oklahoma State University \\ Stillwater, Oklahoma 74078 \\ Email: qi.cheng@okstate.edu
}

\author{
Venkatesh Sarangan \\ Department of Computer Science \\ Oklahoma State University \\ Stillwater, Oklahoma 74078 \\ Email: saranga@cs.okstate.edu
}

\begin{abstract}
Cognitive radio has been proposed as an innovative and effective technology to exploit the efficient reuse of the precious radio spectrum. In spectrum sensing which is a crucial step in cognitive radio networks, an adequate sensing range needs to be defined to avoid the hidden transmitter/receiver problems. Moreover, a secondary user may have unreliable detection of the existent primary users due to multipath fading and/or shadowing. To solve these issues, we propose a cooperative spectrum sensing scheme with adaptive sensing ranges, especially for the hidden receiver problem. We explain the idea of using the adaptive sensing range at a secondary user transmitter and show with the simulation results that cooperative spectrum sensing with adaptive sensing ranges achieves a higher detection accuracy than that with a fixed sensing range.
\end{abstract}

\section{INTRODUCTION}

Recently, cognitive radio (CR) has been proposed as an innovative and effective technology to exploit the efficient reuse of the precious radio spectrum. With the cognitive radio technology, secondary users (SU) who do not hold the license dynamically sense, identify, and then operate in the unused frequency bands. When the primary user wants to use its licensed spectrum again, secondary users quickly vacate the frequency band. Therefore, the overall spectrum utilization can be effectively improved. Since CR networks operate over a set of channels whose availability changes over time, these networks are inherently multi-channel. An appropriate multichannel MAC protocol will have advantages such as reducing interference among the nodes and increasing the capacity of the wireless network significantly.

When we design cognitive radio MAC protocol, there are three possible sources of detection errors that need to be considered [1]: hidden transmitter (node X), hidden receiver (node $Y$ ), and exposed transmitter (node $Z$ ) as shown in Fig.1. These errors are dependent on the transmission range, the sensing range, and the interference range, which are explained for IEEE 802.11 in detail in [6]. Generally, node A cannot distinguish the exposed transmitter and other transmitters when node A conducts spectrum sensing. Therefore, we do not consider the exposed transmitter problem in this paper.

Another challenge associated with $\mathrm{CR}$ networks is the shadowing uncertainty problem [2]. A secondary user may suffer from unexpected multipath fading and/or shadowing when sensing the signal from a primary transmitter. To account for possible losses due to fading, the secondary user must be significantly more sensitive in detecting the primary user's signal. In addition, existing problems in conventional multichannel wireless networks such as the multi-channel hidden terminal problem [3] which is severe in distributed networks still remain unsolved in CR networks.

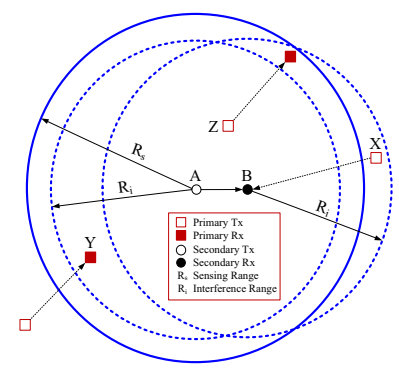

Fig. 1. Spectrum opportunity detection. Node $X$ is the hidden transmitter. Node $\mathrm{Y}$ is the hidden receiver. Node $\mathrm{Z}$ is the exposed transmitter

To solve these problems, many methods have been proposed, among which the cooperative spectrum sensing scheme has been shown to be very effective in tackling the hidden terminal problem [4][5]. Moreover, it has demonstrated increased accuracy in detecting primary users in fading environments. Since a fixed and common sensing range at all nodes is adopted in most of the existing cooperative schemes, the number of unnecessarily detected primary users inevitably increases. That is, some of the primary users within the sensing rage of cooperative nodes do not effect the transmission of the secondary user and vice versa. More details on the related work are provided in Section II.

In this paper, we propose that adaptive sensing ranges should be adopted by cooperative nodes in CR ad-hoc networks. Note that the sensing range can be adjusted by changing the threshold of an energy detector [1]. The main contributions of this paper are: 1) For solving the hidden transmitter problem, local spectrum sensing at the SU receiver with an adaptive sensing range is proposed. 2) For solving the hidden receiver problem, cooperative spectrum sensing at the SU transmitter with cooperative partners using adaptive sensing ranges is proposed. 3) A cooperative sensing MAC protocol using adaptive sensing ranges is proposed. 4) The 
performances of using adaptive sensing ranges as well as using a fixed sensing range at cooperative partners are compared. We demonstrate through simulations that cooperative partners using adaptive sensing ranges can improve the detection accuracy significantly.

\section{PRELIMINARIES}

\section{A. SIR-based Model of IEEE 802.11 Ad-Hoc Networks}

The large scale path loss model is commonly used to describe the radio propagation property in wireless networks [6]. For simplicity, we assume that the transmission power and other radio parameters at all the nodes are the same. This implies that the transmission range of all transmitters is the same. According to this model, the received power at distance $d$ is given by

$$
P_{r}=P_{t} G_{t} G_{r} \frac{h_{t}^{2} h_{r}^{2}}{d^{\alpha}}
$$

where $P_{t}$ is the transmitted power, $h_{t}$ and $h_{r}$ are the heights of the transmitter and receiver antennas respectively, $G_{t}$ and $G_{r}$ are the antenna gains, $d$ is the distance between the transmitter and the receiver. $\alpha$ is the path loss exponent which reflects how fast the signal attenuates. (e.g., 4 in the two-ray ground reflection model and 2 in a free space model).

Successful reception of a packet at the physical layer depends on the signal to interference ratio (SIR) at the receiver. Assume that a transmitter is $d$ meters away from a receiver and at the mean time, an interfering node $r$ meters away from the receiver starts another transmission. Let $P_{r}$ denote the receiving power of the signal from the transmitter and $P_{i}$ denote the power of the interference signal at the receiver. Neglecting the thermal noise, we have [7][8]

$$
S I R=\frac{P_{r}}{P_{i}}=\frac{P_{t} G_{t} G_{r} \frac{h_{t}^{2} h_{r}^{2}}{d^{\alpha}}}{P_{t} G_{t} G_{r} \frac{h_{t}^{2} h_{r}^{2}}{r^{\alpha}}}=\left(\frac{r}{d}\right)^{\alpha}
$$

For a correct reception at the receiver, SIR should be equal to or greater than a capture threshold, $S_{0}$, which in practice is usually set to $10 \mathrm{~dB}$ as in the $802.11 \mathrm{~b}$ specification. That means the interfering nodes must be at least $\sqrt[\alpha]{S_{0}} \times d$ meters away from the receiver for a successful reception. Thus, the interference range, $R_{i}$, is given by [8-10]

$$
R_{i}=\sqrt[\alpha]{S_{0}} \times d=\gamma \times d
$$

where $\gamma$ is defined as $\sqrt[\alpha]{S_{0}}$. Note that $R_{i}$ is proportional to the one-hop distance $d$. In addition, we can define the transmission range and the sensing range [10]. The transmission range, $R_{t}$, is given by

$$
R_{t}=\bar{d}\left(\frac{\bar{P}_{r x}}{P_{R}}\right)^{\frac{1}{\alpha}}
$$

where $\bar{P}_{r x}$ is the reference signal strength as measured at the distance $\bar{d}$ (usually 1 meter) and $P_{R}$ is the reception power threshold. The sensing range, $R_{s}$, is given by

$$
R_{s}=\bar{d}\left(\frac{\bar{P}_{r x}}{P_{C}}\right)^{\frac{1}{\alpha}}
$$

where $P_{C}$ denotes the carrier sensing threshold.

The concepts of the interference range and the transmission range are illustrated in Fig.2. Note that $R_{t}$ and $R_{s}$ are merely radio ranges, which implies that they only apply in the physical layer. However, $R_{i}$ is involved in the SIR model and the comparison of the received power, so it should be managed at the MAC layer as well. Specifically, we can see from Eq.(3) that when $d$ is equal to $R_{t}$, the maximum interference range can be obtained.

\section{B. Problems of IEEE 802.11 DCF in Ad-Hoc Networks}

Virtual Carrier Sensing (VCS) is designed mainly to solve the hidden terminal problem, which involves a basic assumption that all hidden nodes are within the transmission range of the receiver. However, some nodes located in the interference range may still interfere with the receiver. This situation rarely occurs in the infrastructured WLAN because most nodes are within the transmission range of either transmitters or receivers. However, this can become a serious problem in adhoc networks due to the wide distribution of nodes and the multi-hop operation.

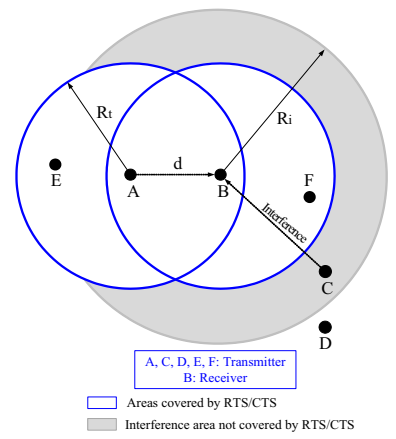

Fig. 2. Interference range $\left(R_{i}\right)$ when $d$ is larger than $1 / \gamma * R_{t}$

For example, in Fig.2, the VCS scheme can effectively prevent nodes $\mathrm{E}$ and $\mathrm{F}$ from initiating an interfering transmission as they can receive RTS/CTS, respectively. Node D cannot make interference at node $\mathrm{B}$ either because this node is out of the interference range of the receiver. However, since node $\mathrm{C}$ within the interference range of node $\mathrm{B}$ cannot receive $\mathrm{RTS} / \mathrm{CTS}$, it can be an interferer to node $\mathrm{B}$ because the ratio of the received signal power from node $\mathrm{A}$ and the interference signal power from node $\mathrm{C}$ is smaller than the capture threshold. Therefore, VCS is not always effective in preventing interference from hidden terminals in ad-hoc networks.

\section{General Concept of Cooperative Spectrum Sensing}

Cooperative spectrum sensing can help solve hidden terminal/shadowing uncertainty problems, mitigating the multipath fading and shadowing effects and improving the detection probability in a heavily shadowed environment [2][4-5]. This is gradually regarded as a key approach in practical implementation of cognitive radio. In general, cooperative spectrum sensing is performed as follows [4]: 1) Each secondary user 
performs local spectrum sensing independently after receiving the request packet from SU Tx node and then makes a binary decision on whether PU Tx is present or not. 2) All of the CRs forward their decisions to SU Tx. 3) SU Tx combines those binary decisions and makes a final decision regarding the absence or presence of the PU in the sensed band.

For example, in Fig.3, cooperative partners (i.e., CP1, CP2, and CP3) perform the local spectrum sensing independently to detect the available spectrum after receiving the request packet from SU Tx. The sensing ranges of cooperative partners are generally all fixed. PU Tx is located inside the sensing ranges of SU Tx and CP1, but not CP2 and CP3. However, SU Tx itself cannot decide if the channel is used by PU Tx due to shadowing. Instead, CP1 can detect the presence of PU Tx and send its binary decisions to SU Tx. After receiving these binary decisions, SU Tx fuses them and infers the absence or presence of PU Tx.

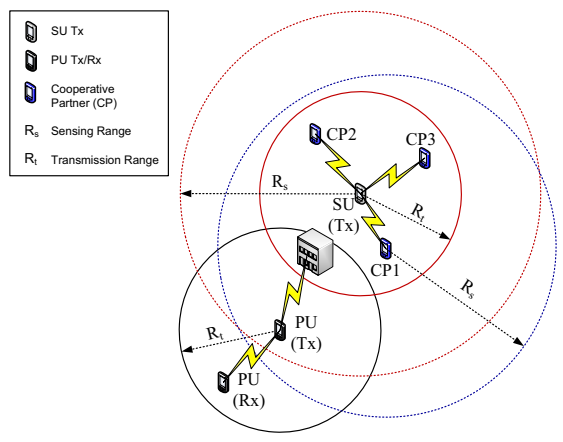

Fig. 3. Cooperative spectrum sensing with a fixed sensing range

\section{SOLUTIONS FOR TWO HIDDEN TERMINAL PROBLEMS}

Our solutions for hidden receiver/transmitter problems are proposed for an 802.11 WLAN based CR ad-hoc networks.

\section{A. Solution for the Hidden Receiver Problem}

To solve the hidden receiver problem, SU transmitter has to detect which PU transmitter sending data to this hidden receiver (i.e., node $\mathrm{Y}$ in Fig.1). Thus, SU transmitter should have the minimum sensing range up to PU transmitter which causes the hidden receiver problem. In Fig.4, the minimum sensing range can be calculated as

$$
R_{s}=\bar{d}\left(\frac{\bar{P}_{r x}}{P_{C}}\right)^{\frac{1}{\alpha}}=R_{i}+R_{t}=\left(1+\sqrt[\alpha]{S_{0}}\right) \times R_{t}
$$

This minimum sensing range is based on the maximum interference range (i.e., $R_{i}$ in Fig.4) of node D, and is the basic requirement for solving the hidden receiver problem. On the other hand, if the sensing range of node A exceeds $R_{s}$, node A may detect unnecessarily some PUs which can operate at the same time with node A. This degrades the efficiency of spectrum utilization.

As we pointed out in the previous section, in a fading environment, spectrum sensing is challenged by the uncertainty due to channel fading. Local spectrum sensing at node
A may be unable to provide the minimum SIR required, with which a secondary user is capable of detecting PU transmitter's signals. To overcome the impact from channel fading, we adopt cooperative sensing with neighbor nodes, which are within the transmission range of node A. Instead of a fixed sensing range, we propose cooperative sensing with adaptive sensing ranges at cooperative partners. We obtain the cooperative partner's adaptive sensing ranges based on the large scale path loss model at the specific locations of the cooperative partners as shown in Fig.4.

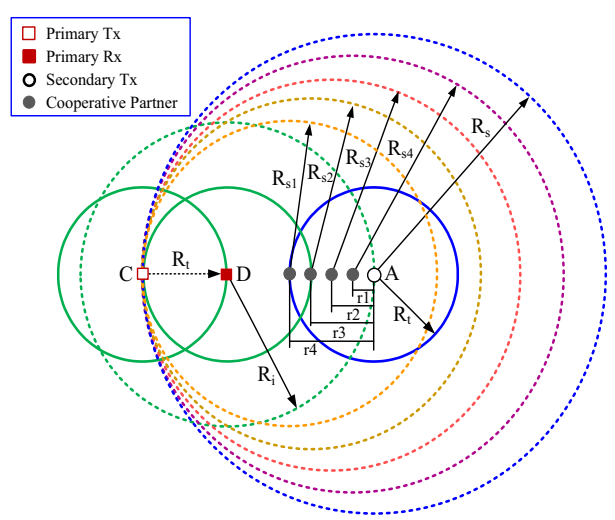

Fig. 4. Cooperative spectrum sensing with adaptive sensing ranges

Upon receiving the request packet from node A, the neighbor nodes calculate their locations, $r_{i}$, based on the received power of the request packets ( $i$ is the node index). From Eq.(1), $r_{i}$ can be obtained by

$$
r_{i}=\sqrt[\alpha]{G_{t} G_{r} h_{t}^{2} h_{r}^{2} \frac{P_{t}}{P_{r}}}=k \times \sqrt[\alpha]{\frac{1}{P_{r}}}
$$

where $k=\sqrt[\alpha]{G_{t} G_{r} h_{t}^{2} h_{r}^{2} P_{t}}$ is a constant. Since SU transmitter's sensing range is known from Eq.(6), we can easily get the cooperative partner's sensing range (CPSR) to detect node C as follows,

$$
C P S R=R_{s}-r_{i}
$$

From Eq.(6) through Eq.(8), the cooperative partner's sensing range at the specific location can be obtained as follows

$$
C P S R=\left[\left(1+\sqrt[\alpha]{S_{0}}\right) \times R_{t}\right]-\left(k \times \sqrt[\alpha]{\frac{1}{P_{r}}}\right)
$$

Note that the cooperative partners using CPSR do not necessarily detect all PUs inside the circle with radius $R_{s}$ because CPSR depends on the locations of the cooperative partners. If, however, the cooperative partners increase their sensing ranges, some PUs outside the circle may be unnecessarily detected. Therefore, there is a tradeoff between the unnecessarily detected PUs and the undetected PUs. In Section $\mathrm{V}$, we compare the performances from this point of view. 


\section{B. Solution for the Hidden Transmitter Problem}

In Fig.1, PU transmitter $\mathrm{X}$ causes the hidden transmitter problem because node $\mathrm{X}$ is outside the sensing range of $\mathrm{SU}$ transmitter A. This implies that when node A plans to send data to node B, node A cannot detect node X's transmission. Thus, the interference will occur at SU receiver B. However, if node $\mathrm{B}$ has the capability to sense the interferer which is within its interference range, this hidden transmitter problem can be solved. Our proposed solution is that during the negotiation stage between SU transmitter and SU receiver, SU receiver also has the local spectrum sensing capability with the sensing range equal to its interference range. In this case, we can calculate this interference range using Eq.(3). Note that $d$ is obtained by Eq.(7).

\section{Proposed Cooperative Sensing MAC Protocol}

Several MAC protocols were proposed for cognitive radio networks in the past [11][12]. However, they do not support cooperative spectrum sensing. We will consider that each node is equipped with a single wireless transceiver. Since our protocol does not require global synchronization, each node in the network is assumed to be aware of a globally common control channel that can be used for exchanging control messages. The proposed MAC protocol consists of three major parts: (1) Listening to the control channel and Initialization with RQT, (2) Channel Sensing, Decision Fusion, and RSP/CRES Exchange over the control channel, (3) DATA/ACK Exchange over data channels. These operations are explained below.

1) Listening to the control channel and Initialization with RQT: When a cognitive radio node is neither transmitting nor receiving, it listens to the control channel. When node A (SU transmitter) wants to transmit data to node $\mathrm{B}$ (SU receiver), node A senses the control channel to see if the control channel is available both physically and virtually. If the control channel is virtually busy because a NAV (Network Allocation Vector) is turned on, the operation is delayed until the NAV has disappeared. When the channel is virtually available, node A senses the control channel physically. If the channel is physically idle, then it waits for DIFS (Distributed InterFrame Space) and transmits an RQT (ReQuesT) packet to cooperative partners which are within its transmission range. If the channel is physically busy, it selects a random backoff counter within a contention window $(\mathrm{CW})$. If the channel is sensed idle after a time slot, it begins the countdown of this random backoff counter; otherwise, the random backoff counter remains frozen. When the counter reaches zero after doing these steps continuously, it transmits an RQT packet. The binary exponential backoff mechanism is employed when collisions occur.

2) Channel Sensing, Decision Fusion, and RSP/CRES Exchange over the control channel: After sending an RQT packet, node A performs its local spectrum sensing with the sensing range the same as $R_{s}$ in Eq.(6). In addition, after receiving an RQT packet, cooperative partners perform the local spectrum sensing independently. Cooperative partners calculate adaptive sensing ranges based on Eq.(9) and sense
PU/SU transmitters with these calculated sensing ranges. Note that cooperative partners cannot distinguish PU transmitters and SU transmitters. Each cooperative partner makes a binary decision based on its local observation and then forwards an RSP (ReSPonse) packet including the sensed channel information to node A after SIFS (Short Inter-Frame Space). Then these cooperative partners set their NAVs. Node B also receives the RQT packet because node $B$ is within node A's transmission range. Accordingly, node B performs the local spectrum sensing with the sensing range the same as node B's interference range. As we explain before, node B's local spectrum sensing is for preventing the hidden transmitter problem. Then node B replies an RSP packet including the sensed channel information to node A after SIFS.

After receiving the RSP packet from node B, neighbor nodes within node B's transmission range set their NAVs. However, neighbor nodes within the cooperative partners' transmission range do not set NAVs after receiving RSPs from cooperative partners. When cooperative partners and node B send RSP packets to node A, they may experience a control channel contention. After acquiring the available channel information from itself, node B, and cooperative partners, node A conducts a decision fusion in which all binary decisions are fused together according to an OR logic and then sends the CRES (Channel REServe) packet including the list of available channels to node B after SIFS.

3) DATA/ACK Exchange over data channels: After sending CRES, node A switches to the chosen data channels. Upon receiving CRES, node $\mathrm{B}$ also switches to the agreed data channels. Node A begins DATA transmission after SIFS. If data are successfully received at node B, node B will send ACK after SIFS. When this transmission is done, node A and $\mathrm{B}$ immediately switch to the control channel. The transmission is considered done after ACK is successfully received. If ACK is not received, node A should go back to the control channel for initialization again.

\section{Performance Evaluation}

We assume that the cooperative partners are distributed uniformly in the transmission range $\left(R_{t}\right)$ of $\mathrm{SU}$ transmitter and all PU transmitters are distributed uniformly over the circle area (radius is $3.78 * R_{t}$ ). In Fig.4, if the cooperative partners sense PUs which are outside the sensing range of SU transmitter, $R_{s}$, these are called the unnecessarily detected $P U s$. Note that this does not occur with the proposed adaptive sensing range. If the cooperative partners do not sense PUs which are inside this range, these are called the undetected $P U s$. Both cases relate to the performance degradation. If the cooperative partners sense PUs which are inside this range, these are called the detected PUs. We consider the cases in which the number of PU transmitters varies from 50 to 100 and the number of cooperative partners varies from 2 to 10 for each specification of the number of PU transmitters.

The performance metric $\rho$ is defined as follows,

$$
\rho=\frac{\text { Undetected PUs }+ \text { Unnecessarily Detected PUs }}{\text { Detected PUs }}
$$


Thus, the smaller $\rho$ represents the better performance. For comparison purpose, conventional cooperative sensing with the fixed sensing range is also evaluated. In this scheme, all nodes use the same sensing range and it is set to $R_{s}$ as in Eq.(6). This is because if the fixed sensing range is smaller than $R_{s}$, then the cooperative partnes may generate more undetected PUs. On the other hand, if the fixed sensing range is larger than $R_{s}$, then the cooperative partners may generate more unnecessarily detected PUs. For simplicity, we assume $R_{s}$ is $2.78 \times R_{t}$ for a two-ray ground reflection model ( $\alpha$ is equal to 4 ) when the capture threshold $\left(S_{0}\right)$ is $10 \mathrm{~dB}$.

Compared with the scheme using a fix sensing range, the cooperative partners involved in the scheme with adaptive sensing ranges have to calculate their individual sensing ranges based on Eq.(9). This increased complexity is not significant in practice, however, due to widely available high performance hardware.

From Fig.5 and Fig.6, we can see that the scheme with the adaptive sensing range has smaller $\rho$ value than that with the fixed sensing range regardless of the number of PUs. As the number of cooperative partners increases, the performance of using adaptive sensing ranges is much better than that of using the fixed sensing range at the cooperative partner. This is because the former scheme does not generate the unnecessarily detected PUs as the cooperative partners do not sense PUs which are outside of the sensing range of SU transmitter. That means the number of unnecessarily detected PUs is always zero. When the number of cooperative partners increases, the number of undetected PUs decreases and the number of detected PUs increases, thus $\rho$ decreases. On the other hand, as the number of cooperative partners using the fixed sensing range increases, the number of unnecessarily detected PUs increases very fast, thus $\rho$ generally increases. As a result, cooperative sensing with our proposed adaptive sensing range is very effective in achieving a higher detection accuracy than that with a fixed sensing range.

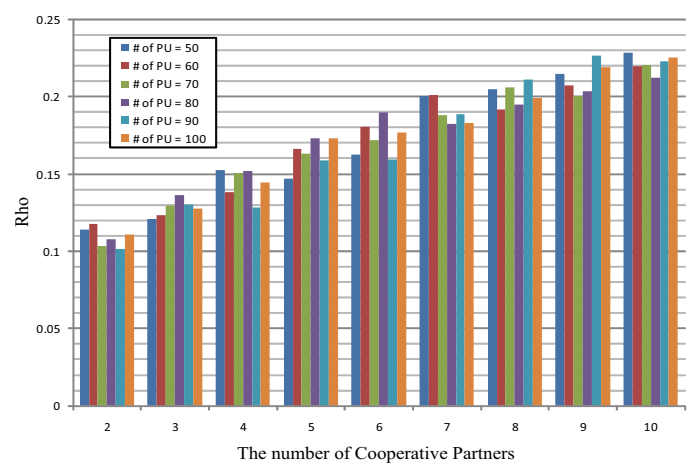

Fig. 5. Performance of Cooperative sensing with the fixed sensing range

\section{CONClusions}

Cognitive radio is an innovative technology to improve the efficiency of the radio spectrum utilization. Cooperative spectrum sensing plays a key role to increase greatly the probability of detection in a channel fading environment. In

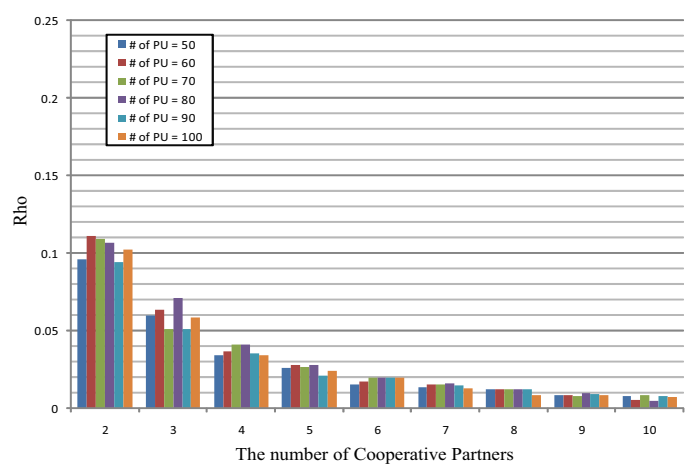

Fig. 6. Performance of Cooperative sensing with adaptive sensing ranges

this paper, we have proposed the solutions for the hidden transmitter and receiver problems in ad-hoc networks and shown that the cooperative spectrum sensing with adaptive sensing ranges outperforms that with a fixed sensing range. Cooperative sensing using our proposed adaptive sensing range is very effective in solving the hidden receiver problem.

Multi-channel MAC protocols have advantages such as reduced interference among the nodes and the increased capacity of wireless networks significantly by operating over multiple channels even if each node only occupies one channel at a time. In our future work, we will investigate and design an effective cognitive multi-channel MAC protocol combined with cooperative sensing using adaptive sensing ranges proposed in this paper.

\section{REFERENCES}

[1] W. Ren, Q. Zhao, and A. Swami, "Power Control in Cognitive Radio Netwroks: How to Cross a Multi-Lane Highway," IEEE Journal on Selected Area in Communications, vol. 27, no. 7, pp. 1283-1296, 2009.

[2] I. F. Akyildiz, W.-Y. Lee, M. C. Vuran, and S. Mohanty, "NeXt Generation/dynamic spectrum access/cognitive radio wireless networks: a survey," Computer Networks, vol. 50, pp. 2127-2159, 2006.

[3] J. So and N. Vaidya, "Multi-Channel MAC for Ad Hoc Networks: Handling Multi-Channel Hidden Terminals Using a Single Transceiver," ACM MobiHoc, pp. 222-233, 2004.

[4] K. B. Letaief and W. Zhang, "Cooperative Communications for Cognitive Raio Networks," Proc. IEEE, vol. 97, no. 5, pp. 878-893, May, 2009.

[5] A. Ghasemi and E. S. Sousa, "Opportunistic Spectrum Access in Fading Channels Through Coolaboratieve Sensing," IEEE Journal of Communications, vol. 2, no. 2, 2007.

[6] P. Gupta and P. R. Kumar, "The Capacity of Wireless Network," IEEE Trans. on Information Theory, vol. 46, no. 2, pp. 388-404, March, 2000.

[7] F. Ye, S. Yi, and B. Sikdar, "Improving Spatial Reuse of IEEE 802.11 Mesh Networks with Enhanced Physical Carrier Sensing," IEEE Communicaitions Society GLOBECOM, 2003.

[8] K. Xu, M. Gerla, and S. Bae, "Effectiveness of RTS/CTS handshake in IEEE 802.11 based Ad Hoc Networks," Ad Hoc Netwroks 1 (ELSEVIER), pp. 107-123, 2003.

[9] K. Xu, M. Gerla, and S. Bae, "How effective is the IEEE 802.11 RTS/CTS Handshake in Ad Hoc Networks?" IEEE Communications Society GLOBECOME, 2002.

[10] J. Zhu, X. Guo, L.L. Yang, and W. S. Conner, "Leveraging Spatial Reuse in 802.11 Mesh Networks with Enhanced Physical Carrier sensing," IEEE International Conference on Communications, vol. 7, pp. 40044011, 2004.

[11] A.C.-C. Hsu, D.S.L. Wei, and C.-C.J. Kuo, "A Cognitive MAC protocol Using Statistical Channel Allocation for Wireless Ad-hoc Networks, IEEE WCNC, pp. 105-110, 2007.

[12] M. Thoppian, S. Venkatesan, R. Prakash, "CSMA-Based MAC Protoco for Cognitive Radio Networks,", IEEE WoWMoM, pp. 1-8, 2007. 